\title{
Does dietary salt induce autoimmunity?
}

Cell Research (2013) 23:872-873. doi:10.1038/cr.2013.65; published online 7 May 2013

Two recent publications suggest that dietary salt may polarize TH17 cells and therefore increase the risk of developing autoimmune disease. Where low salt diets can readily be tested for their therapeutic effects in autoimmune disease, more work is needed to connect dietary salts with the development of immunopathology.

Sodium represents the most abundant cation in the extracellular fluid, where it performs a plethora of biological processes ranging from establishment of cellular membrane potential to osmotic balance. Although sodium is an essential nutrient, in recent years the intake of sodium derived from salt-rich diets across the globe has dramatically increased with numerous detrimental effects being projected including cardiovascular complications and hypertension [1]. Two recent publications postulate a new set of dangers associated with this alarming trend, and hypothesize that rising dietary salt intake may represent a long sought-after link to the ever-increasing prevalence of autoimmunity in the developed world $[2,3]$. Both publications outline a mechanism by which increased salt in our diets can render T cells more receptive to IL-23 signaling, a cytokine historically linked to a number of autoimmune diseases including multiple sclerosis (MS), inflammatory bowel disease (IBD), rheumatoid arthritis (RA) and psoriasis.

IL-23 is a cytokine like few others, in that its requirement for induction of proinflammatory autoimmune disease states in animal models is seemingly absolute [4]. Both manuscripts describe a role of the serum glucocorticoid kinase
1 (SGK-1) in regulating the expression of IL-23R by T cells. Whilst SGK-1 is not essential for $\mathrm{T}_{\mathrm{H}} 17$ polarization per se, Wu et al. [3] unravel a $\mathrm{T}_{\mathrm{H}} 17$-intrinsic transcriptional network in which they describe SGK-1-mediated deactivation of Foxo1, a transcriptional repressor of IL-23R expression using a combination of genetic approaches. $\mathrm{NaCl}$ directly upregulates SGK-1 expression, which in turn leads to Foxo1 phosphorylation and its subsequent inability to inhibit ROR $\gamma \mathrm{t}$-mediated transcription of the $i l 23 r$ locus [3]. When excess $\mathrm{NaCl}$ was added to tissue cultures to promote $\mathrm{T}_{\mathrm{H}} 17$ polarization, expression of IL-17A was enhanced. This finding was also found in human $\mathrm{T}$ cells polarized to produce IL17A [2]. In turn, when mice were kept on high-salt diets, they were reported to be hypersusceptible to experimental autoimmune encephalomyelitis (EAE), the most pervasive animal model of MS. Despite a proposed mechanism centered on IL-23R transcriptional regulation, genes encoding IL17A, IL-17F, ROR $\gamma \mathrm{t}$ and IL-23R all dramatically increased in the absence of any exogenous IL-23 or other $\mathrm{T}_{\mathrm{H}} 17$-polarizing cytokines. Given the inability of $\mathrm{T}$ cells to express functional IL-23, it seems that $\mathrm{NaCl}$ can partially function itself as a polarizing cell culture reagent in this setting. This notion is supported by their additional in vivo finding that even naïve mice show significantly increased numbers of Th17 cells in the lamina propria after being placed on a high-salt diet.

Based on data generated by the world health organization (WHO), a direct correlation between salt intake and prevalence of MS is not apparent in the broad sense [5]. Countries such as China and Japan, which traditionally have the highest salt intake (confirmed in a large number of studies) due to generous addition of salt during cooking and dietary regulars such as soy and miso, also show some of the lowest rates of MS prevalence in the population $(<0.5$ per 100000 of the population) [6]. Conversely, the United States of America and the United Kingdom, which both recorded an MS prevalence of $>100$ per 100000 of the population, have relatively low salt intakes compared to these Asian countries. A simple 'one-toone' overlap was of course unlikely, but one cannot exclude the possibility that for individuals predisposed to autoimmunity through various susceptibility loci, increased salt intake may in fact aggravate their clinical symptoms. This is of course a very attractive concept, as some benefit to patients may be realized at minimal expense and with the greatest of ease simply by avoiding foods rich in salt content.

One major question now is the physiological underpinning of their findings. Increased dietary salt intake does not render higher concentrations of sodium in the blood or lymph nodes, where $\mathrm{T}$ cells usually dwell. The authors propose that higher salt concentrations might alter the gut environment where elevated levels of salt might be sensed. This is an interesting notion to be addressed in future studies. Also, is this phenomenon specific to $\mathrm{NaCl}$ or might elevated IL-17 expression be a response to the hyperphysiological concentrations of other salts in vitro? The authors discovered a fascinating phenomenon and add interesting data to the impact of diet and the gut environment on the immune system, 
but it is early days and the impact of dietary salts on autoimmunity and in particular on MS requires controlled clinical trials. Given that a low-salt diet poses no safety concerns, such studies can be initiated without delay.

The papers mentioned above focus primarily on $\mathrm{CD}^{+} \mathrm{T}$ cells, but other members of the innate immune system represent highly IL-23-responsive cell types. Dietary intake has been shown to have a direct effect on the construction of our immune systems in the gut, and that microflora are heavily influenced by our choice of diet [7]. We have evolved with salt in our diets and as such the evolution of the immune system in the context of salt intake is an attractive concept. IL-23R expression is known to be highest in innate lymphoid cells (ILCs), which line our intestine and populate gut-associated lymphoid tissue (GALT) [8, 9]. One could argue that these cells would be in the firing line of food highly concentrated with salt. Is dietary salt intake required for their maintained expression of IL-23R on ILCs? Would a salt-free diet render mice more susceptible to gut-dwelling bacteria, or even resistant to EAE? These data are certainly 'food for thought'.

Andrew L Croxford ${ }^{1}$, Ari Waisman ${ }^{2}$, Burkhard Becher ${ }^{1}$

${ }^{1}$ Institute of experimental Immunology, University of Zurich, Zurich, Switzerland; ' Institute for Molecular Medicine, University Medical Center of the Johannes-Gutenberg University of Mainz, Germany

Correspondence: Burkhard Becher

E-mail: becher@immunology.uzh.ch

\section{References}

1 Brown IJ, Tzoulaki I, Candeias V, et al. Int J Epidemiol 2009; 38:791-813.

2 Kleinewietfeld M, Manzel A, Titze J, et al. Nature 2013; 496:518-522.

$3 \mathrm{Wu} \mathrm{C}$, Yosef $\mathrm{N}$, Thalhamer T, et al. Nature 2013; 496:513-517.

4 Croxford AL, Mair F, Becher B. Eur J Immunol 2012; 42:2263-2273.

5 Ellliott P, Brown I. World Health Organization. http://www.who.int/dietphysicalactivity/Elliot-brown-2007.pdf Accessed 2013 Apr 15.

6 World Health Organization. cited 2013 Apr 15. Available from: http://www.who.int/ mental_health/neurology/atlas_multiple sclerosis_resources_2008/en/index.html

7 Backhed F, Ley RE, Sonnenburg JL, et al. Science 2005; 307:1915-1920.

8 Buonocore S, Ahern PP, Uhlig HH, et al. Nature 2010; 464:1371-1375.

9 Sonnenberg GF, Monticelli LA, Alenghat T, et al. Science 2012; 336:1321-1325. 